\title{
Sepsis by using Cecal Ligation and Single Puncture Causes Alveolar Space Enlargement in $\mathrm{LPA}_{2}$ Knockout Mice
}

Yutong Zhao ${ }^{1 *}$, Panfeng Fu², Jing Zhao', Rachel K. Mialki ${ }^{1}$, Jianxin Wei', Longshuang Huang ${ }^{2}$, Jerold Chun ${ }^{3}$, Adriana S. Leme ${ }^{1}$, Steven D. Shapiro ${ }^{1}$ and Viswanathan Natarajan ${ }^{2,4}$

${ }^{1}$ Department of Medicine, University of Pittsburgh School of Medicine, Pittsburgh, PA, USA

${ }^{2}$ Department of Pharmacology, University of Illinois at Chicago, Chicago, IL, USA

${ }^{3}$ Department of Molecular Biology, The Scripps Research Institutes, San Diego, CA, USA

${ }^{4}$ Department of Medicine, University of Illinois at Chicago, Chicago, IL, USA

\begin{abstract}
Lysophosphatidic acid (LPA) plays a dual-function in lung inflammatory diseases. LPA receptors contribute to the pathogenesis of asthma, acute lung injury, and fibrosis. Here, we investigate the role of LPA receptor type $2\left(\mathrm{LPA}_{2}\right)$ in sepsis-induced lung inflammation and injury. Sepsis was induced using cecal ligation and single puncture (CLP) with 27 gauge needle. Plasma interleukin-6 (IL-6) and KC levels were elevated in septic wild type and LPA ${ }_{2} /-$ mice, while septic LPA ${ }_{2}-/$ - mice reduces plasma KC, not IL-6 levels, compared to septic wild type mice. Bronchoalveolar lavage (BAL) KC levels increased in septic wild type and $\mathrm{LPA}_{2}-/$ - mice, while the sepsis had no effect on BAL IL-6 levels, protein leak, and inflammatory cell infiltration in the lungs in wild type and $\mathrm{LPA}_{2}-/$ - mice. Hematoxylin and eosin $(\mathrm{H} \& \mathrm{E})$ staining revealed that septic $\mathrm{LPA}_{2}-/$ - mice aggravated alveolar space enlargement. Western blotting analysis of lung tissues demonstrate that the level of cortactin, an F-actin binding protein, was decreased in septic LPA $-/-$ mice, when compared to wild type mice. The level of immunoglobulin $\mathrm{G}(\mathrm{lgG})$ in BAL fluids significantly increased in septic $\mathrm{LPA}_{2} /-$ - mice, when compared to septic wild type mice and sham mice. Furthermore, we found that sham and septic $\mathrm{LPA}_{2}-/-$ mice increased surfactant proteins $\mathrm{B}, \mathrm{C}$, and D (SP-B, SP-C, and SP-D) expression in lungs, while SP-A levels in lungs was decreased in sham and septic $L P A_{2}-/-$ mice. These results suggest $L P A_{2}$ may regulate cortactin and surfactant protein expression in the lung. LPA ${ }_{2}$ and its downstream signaling may play a protective role against sepsis induced emphysema like disease.
\end{abstract}

Keywords: LPA receptor; Sepsis; Alveolar space enlargement; Surfactant protein; Cortactin

Abbreviations: LPA: Lysophosphatidic Acid; CLP: Cecal Ligation and Puncture; IL-6: Interleukin-6; BAL: Bronchoalveolar Lavage; $\mathrm{H}$ \& E: Hematoxylin and Eosin; SP-A-D: Surfactant Protein-A-D; ARDS: Acute Respiratory Distress Syndrome; ELISA: Enzyme-Linked Immunosorbent Assay; IgG: Immunoglobin G

\section{Introduction}

Lysophospholipids have been known as vital components in the organization of membrane structure; however the increasing evidences suggest that lysophospholipids also induce various cellular responses through ligation to their receptors on cell surface. Among the lysophospholipids, lysophosphatidic acid (LPA), a simple biophospholipid, has been detected in various biological fluids, such as plasma [1] and bronchoalveolar larvage (BAL) fluids [2-4]. LPA induces both pro and anti-inflammatory responses in inflammatory lung diseases. Evidence of the pro-inflammatory effect of LPA has been observed by it increasing interleukin-8 (IL-8) production and secretion in lung epithelial cells [5-7]. Intratracheal administration of LPA for 6 $\mathrm{h}$ induces neutrophil infiltration into the alveolar spaces, while at 24 $h$, the effect of LPA on neutrophil infiltration returns to the basal level [5]. Recent studies have shown that intratracheal administration of LPA at $24 \mathrm{~h}$ or intravenous injection of LPA attenuates endotoxin-induced lung inflammation, suggesting that exogenous LPA exhibits an antiinflammatory property [8]. Furthermore, LPA increases IL-13 decoy receptor (IL-13Ra) [9] and IL-33 decoy receptor (sST2) [10] release in human bronchial epithelial cells. In addition to the modulation of inflammatory responses, LPA plays a protective role against lung injury by enhancing lung epithelial barrier integrity and remodeling $[8,11]$.

The biological effects of LPA are through LPA receptors on the cell surface. So far, seven LPA receptors have been cloned [12]. The role of LPA receptors in lung inflammatory diseases have been investigated using LPA receptor deficient mice. LPA receptor $1\left(\mathrm{LPA}_{1}\right)$ mice show a reduction of lung inflammation in murine models of pulmonary fibrosis and acute lung injury [3]. LPA heterozygous knockout mice reduce goblet cell hyperplasia and mucus generation in a murine model of asthma [4]. Down-regulation of $\mathrm{LPA}_{2}$ reduces pathogen induced eosinophil infiltration into airway lumen [4], suggesting that endogenous LPA and its receptors may exhibit pro-inflammatory properties.

Sepsis is a life-threatening systemic disease caused by bacterial infection. Here, we investigate the effect of down-regulating $\mathrm{LPA}_{2}$ in sepsis-induced lung inflammation. This study is the first report to demonstrate that LPA deficient mice show alveolar space enlargement with a reduction of cortactin, an increase in the BAL IgG level, and changes of surfactant proteins in the lungs of a murine model of cecal ligation and puncture (CLP)-induced sepsis. These findings may provide a new therapeutic target against septic lung injury.

\section{Materials and Methods}

$\mathrm{LPA}_{2}$-/- mice - $\mathrm{LPA}_{2}$-/- mice were generated as previously described

*Corresponding author: Yutong Zhao, Department of Medicine, University of Pittsburgh, 3459 Fifth avenue, MUH NW628, Pittsburgh, PA, 15213, USA, Tel: 412 648 9488; E-mail: zhaoy3@upmc.edu

Received May 22, 2011; Accepted June 25, 2012; Published June 30, 2012

Citation: Zhao Y, Fu P, Zhao J, Mialki RK, Wei J, et al. (2012) Sepsis by using Cecal Ligation and Single Puncture Causes Alveolar Space Enlargement in LPA Knockout Mice. J Aller Ther S4:005 doi:10.4172/2155-6121.S4-005

Copyright: (c) 2012 Zhao Y, et al. This is an open-access article distributed under the terms of the Creative Commons Attribution License, which permits unrestricted use, distribution, and reproduction in any medium, provided the original author and source are credited. 
[13]. Mice were bred and housed in a specific pathogen-free barrier facility maintained by the University of Illinois at Chicago Animal Resources Center. The studies reported here conform to the principles outlined by the Animal Welfare Act and the National Institutes of Health guidelines for the care and use of animals in biomedical research. Extract-N-Amp Tissue PCR kit (Sigma Aldrich, S. Louis) was utilized for isolating genomic DNA from mouse tail and amplifying DNA fragments. The primers for LPA, knockout mice were described in previous studies [13].

Sepsis model by CLP - CLP was used to induce sepsis. Briefly, a $3-\mathrm{cm}$ midline laparotomy was made first through the skin and then the cecum with the adjoining intestine was exteriorized and ligated at 0.5 $\mathrm{cm}$ from its end with a 3.0 silk. Then the ligated cecum was punctured with a 27-gauge needle, allowing entrapped fecal material to leak into the normally sterile peritoneal cavity. The cecum was then repositioned in the peritoneal cavity and the abdomen was closed. Sham-operated animals received laparotomy only. After $24 \mathrm{~h}$, plasma, BAL fluids, and lung tissues were collected. After incubation with red cell lysis buffer, cell numbers in BAL fluids were counted by TC10 ${ }^{\mathrm{mm}}$. Automated Cell Counter (Bio-Rad, Hercules, CA) and cell differentiation was performed by cytospin with HEMA3 staining kit (Fisher Scientific, Kalama, MI). $\mathrm{H} \& \mathrm{E}$ staining of lung tissues was performed by histology co-facility in University of Pittsburgh.

Cytokine measurement - BAL fluids and plasma were centrifuged at $500 \mathrm{~g}$ for $10 \mathrm{~min}$ to remove cell debris. IL- 6 and $\mathrm{KC}$ levels were measured with ELISA kits for mouse IL-6 or KC according to the manufacture's instruction (Invitrogen).

\section{Alveolar space measurement}

Chord length, which measures the average distance between alveolar walls, is proportional to the amount of emphysema, as prevously described $[14,15]$. Three randomly selected $\times 10$ fields per slide ( 3 slides /group) were photographed and the images analyzed using Scion Image software (Scion Corporation, Frederick, MD).

RNA isolation and Real-time RT-PCR - Total RNA was isolated from lung tissues using TRIzol ${ }^{\circ}$ reagent (Life Technology, Rockville, MD) according to the manufacturer's instructions. RNA was quantified spectrophotometrically and $1 \mu \mathrm{g}$ of RNA was reversed transcripted using cDNA synthesis kit (Bio-Rad) and Real-time PCR and quantitative PCR were performed to assess expression of the LPA using primers designed based on mouse mRNA sequences. Amplicon expression in each sample was normalized to its $18 \mathrm{~S}$ RNA content. The relative abundance of target mRNA in each sample was calculated as 2 raised to the negative of its threshold cycle value times 106 after being normalized to the abundance of its corresponding 18S [e.g., 2 -(Target Gene Threshold Cycle $) / 2$-(18S Threshold Cycle $) \times 10^{6}$ ]. Primers for PCR: mLPA: Forward: 5'-ATATTCCTGCCGAGATGCTG-3', Reverse: 5'-AAGCTGAGTAACGGGCAGAC-3'; 18S: Forward: 5' -GTAACCCGTTGAACCCCATT-3', Reverse: 5'-CCATCCAATCGGTAGTAGCG-3'

\section{Western blotting}

Equal amounts of protein $(20 \mu \mathrm{g})$ or equal volumes of BAL fluids were subjected to $10 \%$ SDS/PAGE gels, transferred to polyvinylidene difluoride membranes, blocked with $5 \%(\mathrm{w} / \mathrm{v})$ BSA in TBST $(25 \mathrm{mM}$ Tris- $\mathrm{HCl}, \mathrm{pH} 7.4,137 \mathrm{mM} \mathrm{NaCl}$ and $0.1 \%$ Tween-20) for $1 \mathrm{~h}$ and incubated with antibodies (dilute 1:1000) in $5 \%(\mathrm{w} / \mathrm{v})$ BSA in TBST for overnight at $4^{\circ} \mathrm{C}$. The membranes were washed at least three times with TBST at 15 min intervals and then incubated with a rabbit or mouse horseradish peroxidase-conjugated secondary antibody (1: $3,000)$ for $1 \mathrm{~h}$ at room temperature. The membrane was developed with an enhanced chemiluminescence detection system according to Manufacturer's instructions.

\section{Statistical analyses}

All results were subjected to statistical analysis using one-way ANOVA and, where appropriate, analyzed by Student-Newman-Keuls test. Data are expressed as means \pm S.D. of samples $(n=3-7)$ and level of significance was taken as $\mathrm{P}<0.05$.

\section{Results}

Septic wild type and $\mathrm{LPA}_{2}-/$ - mice increase plasma IL-6 levels -
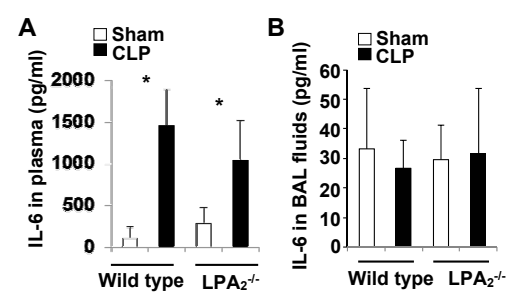
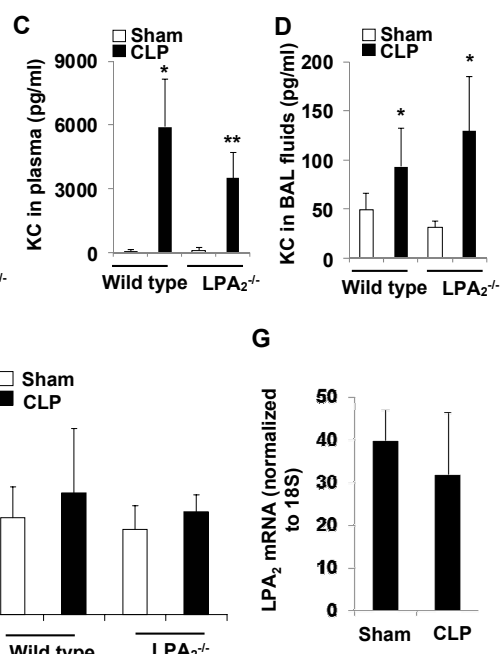

Figure 1: Septic LPA - -/- mice showed similar manner as wild type in plasma IL-6 level, BAL IL-6 level, cell numbers, and protein levels in lungs, except reduction of plasma KC levels $-\mathrm{A}$, C. Wild type and LPA $-/-$ mice were challenged with CLP for $24 \mathrm{~h}$. Plasma IL- 6 and KC levels were measured by a ELISA kits. Data represent mean \pm SD and $n=4-7$. ${ }^{*} p<0.01$ vs sham mice, ${ }^{* *} p<0.05$ vs sham wild type. B, D. Wild type and LPA $-/-$ mice were challenged with CLP for $24 \mathrm{~h}$ and BAL fluids were collected. BAL IL-6 and KC levels were measured by mouse cytokine ELISA kits. Data represent mean \pm SD and $n=4-7$. ${ }^{*} p<0.01$ vs sham mice. E. BAL cell number was accounted. Data represent mean $\pm S D$ and $n=4-7$. F. BAL protein concentration was measured with BSA as series standard. Data represent mean \pm $S D$ and $n=4-7$. G. mouse LPA ${ }_{2}$ RNA expression was examined by Real-time PCR. Data represent mean $\pm S D$ and $n=3-4$. 

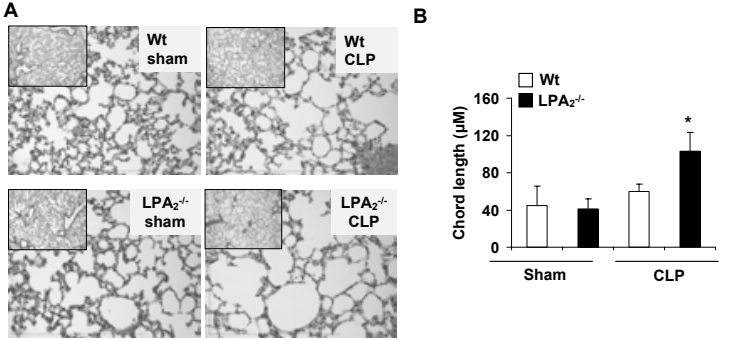

Figure 2: Septic LPA²-/- mice showed enlargement of alveolar spaces - A. Four group mice were sacrificed at $24 \mathrm{~h}$ and lung tissues were fixed and stained with H\&E. B. Chord length of alveolar spaces were measured and quantified by Scion Image software. Data represent mean \pm SD. ${ }^{*} p<0.01$ vs sham wild type sham, and septic LPA $-/-$ mice.

\section{A}

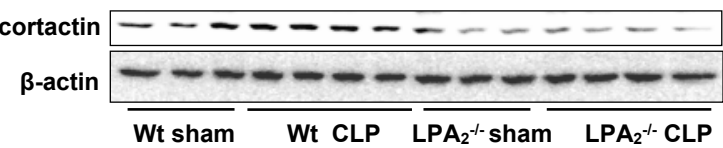

B

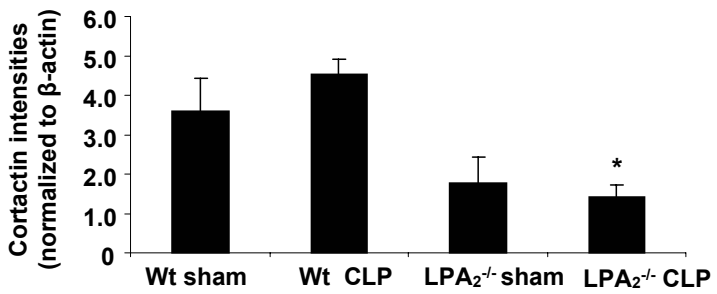

Figure 3: BAL IgG level increased in septic $\mathrm{LPA}_{2}-/-$ mice - Wild type and $L_{P A}-/-$ mice were challenged with CLP for $24 \mathrm{~h}$ and BAL fluids were collected. A. BAL fluids from sham wild type and LPA $-/-$ mice were subjected to 10 $\%$ SDS/PAGE gel and mouse IgG levels were detected by an anti-mouse IgG antibody. B. IgG bands were quantified with ImageJ software. C. BAL fluids from all the four groups were subjected to $10 \%$ SDS/PAGE gel and mouse IgG levels were detected by an anti-mouse IgG antibody. D. IgG bands were quantified with ImageJ software. Data represent mean $\pm \mathrm{SD}$ and $n=5-6$. ${ }^{*} p<$ 0.01 vs sham wild type, sham LPA $_{2}-/-$, and septic wild type mice.

We have shown that $\mathrm{LPA}_{2}$ heterozygous $\left(\mathrm{LPA}_{2}+/-\right)$ mice reduce mucus generation in a mouse model of Th2-dominant inflammatory diseases [4]. Sepsis is a clinical syndrome that complicates severe infection. Treating sepsis during its mild stage is critical because the likelihood of multisystem organ dysfunction increases as it progresses. To investigate the role of LPA in the pathogenesis of sepsis-induced lung injury, we selected a CLP-induced sepsis mouse model. Sepsis was induced by $\mathrm{CLP}$ with a 27 gauge needle. Septic wild type and $\mathrm{LPA}_{2}-/$ - mice survived after a $24 \mathrm{~h}$ period (data not shown). Blood samples were collected and plasma IL-6 and KC levels were determined by ELISA kits. Plasma IL-6 and $\mathrm{KC}$ levels significantly increased in both septic wild type and $\mathrm{LPA}_{2}-$ /- mice when compared to sham mice (Figure 1A-1C), suggesting that CLP induced a systemic inflammatory response.

Plasma KC levels were significantly reduced in septic $\mathrm{LPA}_{2}-$ - mice (Figure 1C) when compared to septic wild type mice, however, plasma IL-6 in septic LPA $-/$ - mice had no statistical difference when compared to septic wild type mice (Figure 1A).

Sepsis increases KC levels, not lung leak and neutrophil influx in wild type and $\mathrm{LPA}_{2}-/$ - mice

To investigate the lung inflammatory responses under sepsis, we measured the BAL IL- 6 and KC levels from septic wild type and $\mathrm{LPA}_{2}$ /- mice. BAL KC levels, but not the BAL IL-6 levels, were increased in both in septic wild type and $\mathrm{LPA}_{2}-/-$ mice, while there was no statistical difference between in the two groups (Figure 1D). These results suggest that LPA / $\mathrm{LPA}_{2}$ axis contributes to KC production in plasma. We further determined whether sepsis induces inflammatory cell infiltration to the lung alveolar spaces. The BAL cell numbers were accounted. As shown in Figure 1E, there was no significant difference in BAL cell numbers within these four groups. Cytospin showed that macrophage is the dominant cell type in BAL fluids in the all four groups (data not shown). In addition, there was no significant change in BAL protein concentration within all the four groups (Figure $1 \mathrm{~F}$ ). To investigate whether CLP affects lung LPA ${ }_{2}$ expression, LPA 2 mRNA expression in lung tissues were examined by Real-time PCR. As shown in Figure $1 G, \mathrm{LPA}_{2}$ mRNA was not changed after CLP. These results suggest that sepsis by CLP with a 27-gauge needle increased KC levels in BAL fluids, but the procedure has limited effects on inflammatory cells influx into lung and lung leak in wild type and $\mathrm{LPA}_{2}-/-$ mice.

\section{Septic $\mathrm{LPA}_{2}$-/- mice show alveolar space enlargement}

Further, we examined the lung histology by H\&E staining. All the four groups did not show significant inflammatory cell influx into the lung alveolar spaces. These data are consistent with the results from cell number accounts (Figure 1E). To estimate the morphological change in response to CLP, we measured the chord length, which measures the average distance between alveolar walls and is proportional to the amount of emphysema. Chord length was not significantly changed in septic wild type mice when compared to sham wild type $(60.2 \pm 7.6$ to $44.6 \pm 21.3 \mu \mathrm{m}, p>0.05$ ). Chord length of sham LPA $-/$ - mice was similar to sham wild type mice ( $44.6 \pm 21.3$ to $40.9 \pm 11.0 \mu \mathrm{m}, p>0.05)$, however, chord length was significantly increased in septic LPA $-/$ - mice when compared to septic wild type mice $(102.9 \pm 20.0$ to $60.2 \pm 7.6 \mu \mathrm{m}$, $41.5 \%, p<0.01$ ) (Figure $2 \mathrm{~A}$ and $2 \mathrm{~B}$ ). These results suggest that $\mathrm{LPA}_{2}$ and its downstream signaling protect against alveolar space enlargement.

\section{$\mathrm{LPA}_{2}-/-$ mice reduced cortactin expression in lung}

To investigate the mechanisms by which septic $\mathrm{LPA}_{2}-/-$ mice show emphysema phenotype, we determined the lung expression levels of an F-actin binding protein, cortactin, since it plays a critical role in maintaining both lung epithelial [11] and endothelial barrier function $[16,17]$. As shown in Figure 3, cortactin levels in lung tissues slightly decreased in sham $\mathrm{LPA}_{2}-/$ - mice, when compared to sham wild type mice, but this was not statistically significant. Cortactin levels in the lungs from septic $\mathrm{LPA}_{2}-/$ - mice were significantly reduced, when compared to sham and septic wild type mice (Figure 3 ). The entirety of the data indicates level of cortactin is less in $\mathrm{LPA}_{2}-/$ - mice, compared to wild type mice. The reduction of cortactin levels in the lungs from septic $\mathrm{LPA}_{2}-/$ - mice may contribute to pathogenesis of emphysema.

\section{Septic $\mathrm{LPA}_{2}$-/- mice increased BAL IgG levels}

IgG levels in BAL fluids are usually very low, whereas BAL IgG levels increase in lung inflammatory diseases. The local BAL IgG production is an index of an increase in invading bacteria or pathogen into the lungs. We measured the BAL IgG levels from sham and septic wild type and $\mathrm{LPA}_{2}-/$ - mice by Western blotting. BAL IgG levels were similar between sham wild type and $\mathrm{LPA}_{2}-/-$ mice (Figure $4 \mathrm{~A}$ and $4 \mathrm{~B}$ ), while BAL IgG levels increased in septic $\mathrm{LPA}_{2}-/$ - mice, but not in septic wild type mice (Figure 4C and 4D). These results suggest that downregulation of $\mathrm{LPA}_{2}$ or its mediated signaling increases IgG levels in BAL fluids in CLP-induced sepsis. 

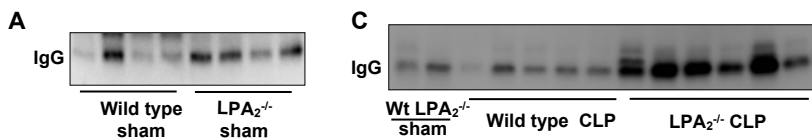

B

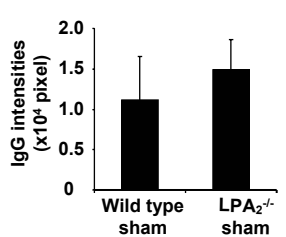

D

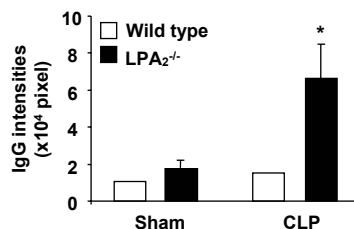

Figure 4: Septic LPA $-/$ - mice reduced cortactin expression in the lungs $-A$ Four group mice were sacrificed at $24 \mathrm{~h}$ and lung tissues were lysed. Equal amount of lung tissue proteins $(20 \mu \mathrm{g})$ were subjected to $10 \%$ SDS/PAGE gel and cortactin levels were determined by Western blotting with antibodies to cortactin and $\beta$-actin. B. Intensities of cortactin and $\beta$-actin bands were analyzed by Image J software. Data represent mean \pm SD and $n=3-4 .{ }^{*} p<$ 0.01 vs sham and septic wild type mice.
A

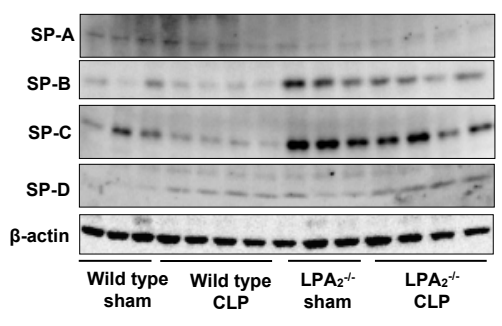

C

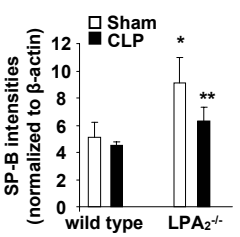

D

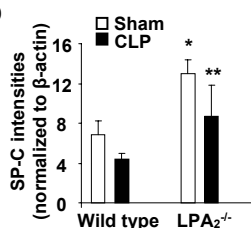

Figure 5: Septic $\mathrm{LPA}_{2}-/$ - mice altered surfactant protein levels in the lung - A Four group mice were sacrificed at $24 \mathrm{~h}$ and lung tissues were lysed. Equal amount of lung tissue proteins $(20 \mu \mathrm{g})$ were subjected to $12 \%$ SDS/PAGE gel and surfactant protein levels were determined by Western blotting with antibodies to surfactant protein A-D and $\beta$-actin. B-E. Intensities of surfactant protein and $\beta$-actin bands were analyzed by ImageJ software. Data represent mean \pm $\mathrm{SD}$ and $\mathrm{n}=3-4$. ${ }^{*} p<0.05$ vs sham wild type mice. ${ }^{*} p<0.05$ vs septic wild type mice.

\section{Septic $\mathrm{LPA}_{2}-/$ - mice changed surfactant proteins levels in the lungs}

In addition to cytoskeleton associated proteins, surfactant proteins play a critical role in maintaining alveolar structure by reducing surface tension and preventing collapse of the lung [18]. The changes of surfactant protein (SP-A, SP-B, SP-C, and SP-D) level in the lungs from the septic wild type and $\mathrm{LPA}_{2}-/-$ mice were examined by Western blotting (Figure 5). SP-A levels in the lungs were reduced in sham and septic $\mathrm{LPA}_{2}-/$ - mice, when compared to sham and septic wild type mice. SP-B levels in lungs were unaltered between sham and septic wild type mice, while SP-B levels in lungs increased in sham and septic $\mathrm{LPA}_{2}-/-$ mice, when compared to sham and septic wild type mice. SP-B levels in lungs from septic $\mathrm{LPA}_{2}-/$ - mice were lower than that from sham $\mathrm{LPA}_{2}-/$ - mice; however it was not statistically significant. SP-C levels in lungs increased in sham and septic $\mathrm{LPA}_{2}^{-/-}$mice, when compared to sham and septic wild type mice. SP-D levels in lungs increased in septic wild type mice and sham $\mathrm{LPA}_{2}-/$ - mice, when compared to sham wild type mice. Furthermore, when compared to septic wild type mice, SP-D levels further increased in septic $\mathrm{LPA}_{2}-/$ - mice. Overall, changes of surfactant protein are more significant between $\mathrm{LPA}_{2}-/$ - and wild type mice, as compared to sham vs. sepsis.

\section{Discussion}

Sepsis is characterized as an inflammatory infection that if not treated promptly can prove to be fatal. Active intracellular signaling and cellular responses are associated with sepsis. The current study focuses on determining the role of a bioactive lysophospholipid receptor, $\mathrm{LPA}_{2}$ in CLP-induced septic lung injury. We found that septic LPA $-/$ - mice show a significant reduction in plasma KC levels, an increase in BAL IgG level, enlargement of alveolar spaces, reduction of cortactin, and changes of surfactant proteins expression in the lungs. Septic LPA /- mice exhibit same manner as septic wild type mice regarding the plasma and BAL IL-6 levels, inflammatory cell infiltration, and protein leak in BAL fluids. These results suggest that $\mathrm{LPA}_{2}$ may protect against emphysema by maintaining alveolar structure.

Among the seven LPA receptors, the roles of $L^{2} A_{1}-3$ in lung inflammatory diseases have been investigated. LPA receptors contribute to pathogenesis of asthma [2,4], fibrosis [19], and acute lung injury [3]. LPA deficient mice reduce intratracheal LPS [3] and bleomycin [19]-induced acute lung injuries. Mucus generation is attenuated in pathogen-induced LPA and LPA heterozygous knockout mice [4]. To investigate the role of $\mathrm{LPA}_{2}$ in the pathological changes of the lungs in sepsis, we generated a murine model of sepsis using CLP with 27-gauge needle. The model demonstrates increases in plasma IL-6 and KC levels and BAL KC levels in septic wild type and $\mathrm{LPA}_{2}-/-$ mice. This suggests that the sepsis model used in this study induces a systemic inflammation, such as BAL KC release in the lungs, however, there is no increase in lung protein leak and inflammatory cell influx. LPA plays a pro-inflammatory role by inducing IL-8 [5-7] and PGE2 [20] release in several cell types, including lung epithelial, endothelial [21], and smooth muscle cells [22]. LPA ${ }_{1-3}$ is involved in LPA-induced activation of transcriptional factors. We have shown that LPA1 contributes to LPS-induced signaling via interaction with LPS co-receptor, CD14 [3]. The role of $\mathrm{LPA}_{2}$ in lung inflammation has been investigated in murine models of asthma [4] and fibrosis (Zhao Y et al. unpublished data). LPA contribute to LPA-induced TGF $\beta$ activation [23] and IL-8 production [6]. $\mathrm{LPA}_{2}$ heterozygous knockout mice reduce mucus generation and eosinophil infiltration into alveolar spaces in the asthma model [4] and lessen inflammation and protein leak in the lung in a bleomycininduced murine model (Zhao Y et al. unpublished data). The current study suggests that $\mathrm{LPA}_{2}$ is involved in plasma $\mathrm{KC}$ production, but not plasma IL- 6 and BAL KC production, in sepsis.

The novel finding in this study is that $\mathrm{LPA}_{2}-/$ - mice exhibit emphysema-like phenotype with reduction of cortactin, increases of BAL IgG, and changes of surfactant protein expression. This is the first report to demonstrate that $\mathrm{LPA}_{2}$ plays a protective role in maintaining alveolar structure.

LPA exhibits an anti-apoptosis via ligation to LPA 2 [24]. To investigate whether the alveolar space enlargement in $\mathrm{LPA}_{2}-/$ - mice is due to an increase in apoptosis, we examined the apoptotic cells in lung tissues by TUNEL assay and Western blotting with a cleaved caspase 3 antibody. There was no significant increase in apoptosis in septic wild type and $\mathrm{LPA}_{2}-$ - - mice, when compared to sham mice (data not shown). Interestingly, the cortactin expression in the lungs from septic $\mathrm{LPA}_{2}$ 
/- mice was significantly reduced. Cortactin, a F-actin binding protein plays a critical role in maintaining cell structure, cell-cell contact, and migration $[25,26]$. Cortactin activation is induced by LPA and mediates LPA-induced lung epithelial cell migration [11]. Cortactin deficiency increase vascular permeability [27]. The loss of cortactin might contribute to pathogenesis of alveolar space enlargement in septic $\mathrm{LPA}_{2}-/$ - mice. The current study demonstrates the reduction of cortactin has no association with alveolar permeability in septic $\mathrm{LPA}_{2}-/$ - mice. The disparity may be due to the levels of cortactin in the different systems. The current study demonstrated that CLP-challenged $\mathrm{LPA}_{2}-/-$ mice partially reduce cortactin levels $(\sim 52 \%$ reduction when compared to wild type). This partial reduction of cortactin may not be sufficient to induce permeability and cell death, while it may contribute to cytoskeleton rearrangement thus causing an increase in alveolar spaces.

Furthermore, we found that $\mathrm{LPA}_{2} / /$ - mice increase the BAL IgG level and change the surfactant protein expression in the lungs. Serum IgG is a biomarker for emphysema [28]. The local BAL IgG production indicates an increase in invading bacteria into the lungs. The increases in BAL IgG in LPA $-/$ - mice are not likely from circulation, since there is no endothelial and epithelial barrier disruption in the current model. Intratracheal injection of IgG immune complex induces rat lung injury [29]. These results, at least, in part, suggest that $\mathrm{LPA}_{2}-/-$ mice increase bacterial invasion into the lungs after CLP and that the lack of the LPA/LPA 2 axis might promote bacterial invasionmediated airspace enlargement. Changes of surfactant proteins are associated with respiratory failures [30-32]. For example, serum SP-A and SP-D increase in patients with septic acute respiratory distress syndrome (ARDS) [33] and serum SP-A and SP-B increase in patients with acute respiratory failure [31,32]. A significant decrease in SP-A, SP-B, and SP-C levels in septic adult sheep has been observed [34]. The role of changes of surfactant protein expression in the pathogenesis of alveolar space enlargement in septic $\mathrm{LPA}_{2}-/$ - mice is not clear, whereas LPA2 and its mediated downstream signaling regulates surfactant protein expression in sepsis. Increased SP-D plays a protective role in the development of emphysema, in part by preventing alveolar cell death [31]. Here, the increases in surfactant proteins in sham and CLP-challenged $\mathrm{LPA}_{2}-/-$ mice might be a negative feedback loop in providing host defense for the lungs. The reduction of $\mathrm{LPA}_{2}$ and cortactin levels in lungs might be developed as biomarkers for the high risk of emphysema. Future studies will focus on the mechanisms by which LPA and LPA regulate cortactin and surfactant protein expression and stability.

\section{Acknowledgements}

All authors have read the journal's policy on disclosure of potential conflicts of interest. The authors report no financial or other conflict of interest relevant to the subject of this article. This study was supported by National Institutes of Health RO1 091916 (to Y.Z.), HLR37 079396 (to V.N.), and American Heart Association Scientist Development Grant 12SDG9050005 (to J.Z.). We thank Drs. Mallampalli, R.K. and Reilly, J.J. for the discussion.

\section{References}

1. Xu Y, Shen Z, Wiper DW, Wu M, Morton RE, et al. (1998) Lysophosphatidic acid as a potential biomarker for ovarian and other gynecologic cancers. JAMA 280: 719-723.

2. Georas SN, Berdyshev E, Hubbard W, Gorshkova IA, Usatyuk PV, et al. (2007) Lysophosphatidic acid is detectable in human bronchoalveolar lavage fluids at baseline and increased after segmental allergen challenge. Clin Exp Allergy 37: $311-322$

3. Zhao J, He D, Su Y, Berdyshev E, Chun J, et al. (2011) Lysophosphatidic acid receptor 1 modulates lipopolysaccharide-induced inflammation in alveolar epithelial cells and murine lungs. Am J Physiol Lung Cell Mol Physiol 301: L547- L556.
4. Zhao Y, Tong J, He D, Pendyala S, Evgeny B, et al. (2009) Role of lysophosphatidic acid receptor LPA2 in the development of allergic airway inflammation in a murine model of asthma. Respir Res 10: 114.

5. Cummings R, Zhao Y, Jacoby D, Spannhake EW, Ohba M, et al. (2004) Protein kinase Cdelta mediates lysophosphatidic acid-induced NF-kappaB activation and interleukin-8 secretion in human bronchial epithelial cells. J Biol Chem 279 41085-41094.

6. Saatian B, Zhao Y, He D, Georas SN, Watkins T, et al. (2006) Transcriptional regulation of lysophosphatidic acid-induced interleukin-8 expression and secretion by $\mathrm{p} 38$ MAPK and JNK in human bronchial epithelial cells. Biochem J 393: 657-668.

7. Zhao Y, Usatyuk PV, Cummings R, Saatian B, He D, et al. (2005) Lipid phosphate phosphatase-1 regulates lysophosphatidic acid-induced calcium release, NF-kappaB activation and interleukin-8 secretion in human bronchial epithelial cells. Biochem J 385: 493-502.

8. He D, Su Y, Usatyuk PV, Spannhake EW, Kogut P, et al. (2009) Lysophosphatidic acid enhances pulmonary epithelial barrier integrity and protects endotoxininduced epithelial barrier disruption and lung injury. J Biol Chem 284: 24123 24132.

9. Zhao Y, He D, Zhao J, Wang L, Leff AR, et al. (2007) Lysophosphatidic acid induces interleukin-13 (IL-13) receptor alpha2 expression and inhibits IL-13 signaling in primary human bronchial epithelial cells. J Biol Chem 282: 1017210179.

10. Zhao J, Chen Q, Li H, Myerburg M, Spannhake EW, et al. (2012) Lysophosphatidic acid increases soluble ST2 expression in mouse lung and human bronchial epithelial cells. Cell Signal. 24: 77-85

11. Zhao J, He D, Berdyshev E, Zhong M, Salgia R, et al. (2011) Autotaxin induces lung epithelial cell migration through lysoPLD activity-dependent and -independent pathways. Biochem J 439: 45-55.

12. Zhao Y, Natarajan V (2009) Lysophosphatidic acid signaling in airway epithelium: role in airway inflammation and remodeling. Cell Signal 21: 367377.

13. Contos JJ, Ishii I, Fukushima N, Kingsbury MA, YeX, etal. (2002) Characterization of Ipa(2) (Edg4) and Ipa(1)/lpa(2) (Edg2/Edg4) lysophosphatidic acid receptor knockout mice: signaling deficits without obvious phenotypic abnormality attributable to Ipa(2). Mol Cell Biol 22: 6921-6929.

14. Hautamaki RD, Kobayashi DK, Senior RM, Shapiro SD (1997) Requirement for macrophage elastase for cigarette smoke-induced emphysema in mice. Science 277: 2002-2004

15. Xiong Z, Leme AS, Ray P, Shapiro SD, Lee JS (2011) CX3CR1+ lung mononuclear phagocytes spatially confined to the interstitium produce TNFalpha and IL-6 and promote cigarette smoke-induced emphysema. J Immunol 186: $3206-3214$

16. Dudek SM, Jacobson JR, Chiang ET, Birukov KG, Wang P (2004) Pulmonary endothelial cell barrier enhancement by sphingosine 1-phosphate: roles for cortactin and myosin light chain kinase. J Biol Chem 279: 24692-24700.

17. Dudek SM, Birukov KG, Zhan X, Garcia JG (2002) Novel interaction of cortactin with endothelial cell myosin light chain kinase. Biochem Biophys Res Commun 298: $511-519$

18. Chander A, Fisher AB (1990) Regulation of lung surfactant secretion. Am J Physiol 258: L241- L253.

19. Tager AM, LaCamera P, Shea BS, Campanella GS, Selman M, et al. (2008) The lysophosphatidic acid receptor LPA1 links pulmonary fibrosis to lung injury by mediating fibroblast recruitment and vascular leak. Nat Med 14: 45-54.

20. He D, Natarajan V, Stern R, Gorshkova IA, Solway J (2008) Lysophosphatidic acid-induced transactivation of epidermal growth factor receptor regulates cyclo-oxygenase-2 expression and prostaglandin E(2) release via C/EBPbeta in human bronchial epithelial cells. Biochem J 412: 153-162.

21. Shimada H, Rajagopalan LE (2010) Rho-kinase mediates lysophosphatidic acid-induced IL-8 and MCP-1 production via p38 and JNK pathways in human endothelial cells. FEBS Lett 584: 2827-2832.

22. Liu JP, Komachi M, Tomura H, Mogi C, Damirin A, et al. (2010) Ovarian cance $\mathrm{G}$ proteincoupled receptor 1-dependent and -independent vascular actions to acidic $\mathrm{pH}$ in human aortic smooth muscle cells. Am J Physiol Heart Circ Physiol 299: $\mathrm{H} 731-\mathrm{H} 742$ 
Citation: Zhao Y, Fu P, Zhao J, Mialki RK, Wei J, et al. (2012) Sepsis by using Cecal Ligation and Single Puncture Causes Alveolar Space Enlargement in LPA ${ }_{2}$ Knockout Mice. J Aller Ther S4:005 doi:10.4172/2155-6121.S4-005

23. Xu MY, Porte J, KnoxAJ, Weinreb PH, Maher TM, et al. (2009) Lysophosphatidic acid induces alphavbeta6 integrin-mediated TGF-beta activation via the LPA2 receptor and the small G protein G alpha(q). Am J Pathol 174: 1264-1279.

24. Hsia CC, Hyde DM, Ochs M, Weibel ER (2010) An official research policy statement of the American Thoracic Society/European Respiratory Society: standards for quantitative assessment of lung structure. Am J Respir Crit Care Med 181: 394-418.

25. Deng W, Shuyu E, Tsukahara R, Valentine WJ, Durgam G, et al. (2007) The lysophosphatidic acid type 2 receptor is required for protection against radiation-induced intestinal injury. Gastroenterology 132: 1834-1851.

26. Weaver AM, Karginov AV, Kinley AW, Weed SA, Li Y, et al. (2001) Cortactin promotes and stabilizes Arp2/3-induced actin filament network formation. Curr Biol 11: 370-374.

27. Weed SA, Parsons JT (2001) Cortactin: coupling membrane dynamics to cortical actin assembly. Oncogene 20: 6418-6434.

28. Schnoor M, Lai FP, Zarbock A, Klaver R, Polaschegg C, et al. (2011) Cortactin deficiency is associated with reduced neutrophil recruitment but increased vascular permeability in vivo. J Exp Med 208: 1721-1735.
29. Falk GA, Siskind GW, Smith JP Jr (1970) Immunoglobulin elevations in the serum of patients with chronic bronchitis and emphysema. J Immunol 105: 1559-1562.

30. Shanley TP, Warner RL, Crouch LD, Dietsch GN, Clark DL, et al. (1998) Requirements for alpha d in IgG immune complex-induced rat lung injury. J Immunol 160: 1014-1020.

31. Lewis JF, Jobe AH (1993) Surfactant and the adult respiratory distress syndrome. Am Rev Respir Dis 147: 218-233.

32. Bersten AD, Hunt T, Nicholas TE, Doyle IR (2001) Elevated plasma surfactant protein-B predicts development of acute respiratory distress syndrome in patients with acute respiratory failure. Am J Respir Crit Care Med 164: 648-652.

33. Doyle IR, Bersten AD, Nicholas TE (1997) Surfactant proteins-A and -B are elevated in plasma of patients with acute respiratory failure. Am J Respir Crit Care Med 156: 1217-1229.

34. Endo S, Sato N, Nakae H, Yamada Y, Makabe H, et al. (2002) Surfactant protein A and D (SP-A, AP-D) levels in patients with septic ARDS. Res Commun Mol Pathol Pharmacol 111: 245-251.
This article was originally published in a special issue, Lung Disorders/Injury handled by Editor(s). Dr. Yutong Zhao, University of Pittsburgh, USA 